\title{
Acute ingestion of catechin-rich green tea improves postprandial glucose status and increases serum thioredoxin concentrations in postmenopausal women
}

\author{
Masaki Takahashi ${ }^{1}$, Masashi Miyashita ${ }^{2 *}$, Katsuhiko Suzuki ${ }^{1}$, Seong-ryu Bae ${ }^{3}$, Hyeon-Ki Kim ${ }^{3}$, \\ Takuya Wakisaka ${ }^{4}$, Yuji Matsui ${ }^{4}$, Masao Takeshita ${ }^{4}$ and Koichi Yasunaga ${ }^{4}$ \\ ${ }^{1}$ Faculty of Sport Sciences, Waseda University, 2-579-15 Mikajima, Tokorozawa, Saitama 359-1192, Japan \\ ${ }^{2}$ Department of Health and Sports Sciences, Faculty of Education, Tokyo Gakugei University, 4-1-1 Nukuikitamachi, \\ Koganei, Tokyo 184-8501, Japan \\ ${ }^{3}$ Graduate School of Sport Sciences, Waseda University, 2-579-15 Mikajima, Tokorozawa, Saitama 359-1192, Japan \\ ${ }^{4}$ Health Care Food Research, Kao Corporation, 2-1-3, Bunka Sumida-ku, Tokyo 131-8501, Japan
}

(Submitted 27 February 2014 - Final revision received 15 July 2014 - Accepted 23 July 2014 - First published online 18 September 2014)

\section{Abstract}

Elevated postprandial hyperglycaemia and oxidative stress increase the risks of type 2 diabetes and CVD. Green tea catechin possesses antidiabetic properties and antioxidant capacity. In the present study, we examined the acute and continuous effects of ingestion of catechin-rich green tea on postprandial hyperglycaemia and oxidative stress in healthy postmenopausal women. Participants were randomly assigned into the placebo ( $\mathrm{P}, n$ 11) or green tea (GT, $n$ 11) group. The GT group consumed a catechin-rich green tea (catechins $615 \mathrm{mg} /$ $350 \mathrm{ml}$ ) beverage per $\mathrm{d}$ for 4 weeks. The P group consumed a placebo (catechins $92 \mathrm{mg} / 350 \mathrm{ml}$ ) beverage per $\mathrm{d}$ for $4 \mathrm{weeks}$. At baseline and after 4 weeks, participants of each group consumed their designated beverages with breakfast and consumed lunch $3 \mathrm{~h}$ after breakfast. Venous blood samples were collected in the fasted state $(0 \mathrm{~h})$ and at 2, 4 and $6 \mathrm{~h}$ after breakfast. Postprandial glucose concentrations were $3 \%$ lower in the GT group than in the P group (three-factor ANOVA, group $\times$ time interaction, $P<0.05$ ). Serum concentrations of the derivatives of reactive oxygen metabolites increased after meals $(P<0 \cdot 05)$, but no effect of catechin-rich green tea intake was observed. Conversely, serum postprandial thioredoxin concentrations were 5\% higher in the GT group than in the P group (three-factor ANOVA, group $\times$ time interaction, $P<0 \cdot 05)$. These findings indicate that an acute ingestion of catechin-rich green tea has beneficial effects on postprandial glucose and redox homeostasis in postmenopausal women.

Key words: Catechins: Postprandial glucose: Postprandial oxidative stress: Postprandial antioxidant capacity

Green tea polyphenols, catechins in particular, have beneficial effects that prevent the development of diabetes and CVD. In fact, some epidemiological studies have reported that regular green tea consumption is associated with reduced CVDrelated mortality ${ }^{(1-3)}$. Although a previous meta-analysis has found that regular consumption of green tea was associated with a significant reduction in fasting glucose ${ }^{(4)}$ and insulin concentrations $^{(5)}$, limited evidence is available regarding the effect of green tea on postprandial hyperglycaemia, which is an independent risk factor for CVD and type 2 diabetes mellitus ${ }^{(6,7)}$. Therefore, it is necessary to examine the effects of green tea consumption on postprandial hyperglycaemia.

Human studies have demonstrated that postprandial hyperglycaemia and hyperlipidaemia are positively correlated with oxidant production ${ }^{(8-10)}$. Moreover, a previous study ${ }^{(11)}$ has shown that intake of a high-lipid diet resulted in greater postprandial oxidative stress than intake of a carbohydrate diet with the same energy content. Elevated postprandial oxidative stress plays a role in the development of atherosclerosis through endothelial dysfunction ${ }^{(12,13)}$. Thus, antioxidant supplementation may be key in preventing postprandial oxidative stress.

Many investigations have highlighted the effects of antioxidant supplementation, including green tea, on postprandial oxidative stress ${ }^{(13-15)}$. The antioxidant effects of green tea are partly attributed to catechins. Some studies ${ }^{(16-18)}$ have reported that green tea polyphenols, specifically catechins, ameliorate the elevation of fasting oxidative stress. However, as there is no conclusive evidence for the effect of green tea on postprandial oxidative stress, further research is needed

Abbreviations: d-ROM, derivatives of reactive oxygen metabolites; GT, green tea; P, placebo; TRX, thioredoxin.

*Corresponding author: Dr M. Miyashita, fax +81 42329 7622, email masashi@u-gakugei.ac.jp 
to determine the role of catechins on postprandial oxidative stress in postmenopausal women who are likely to show increased oxidative stress ${ }^{(19)}$. To date, no information is available regarding the effect of green tea on postprandial oxidative stress in postmenopausal women. From the viewpoint of ageing and menopause, interventions that modulate oxidative stress are needed to examine this age group. The purpose of the present study was to examine the acute and continuous effects of the ingestion of catechin-rich green tea on postprandial glucose and oxidative stress in postmenopausal women. We hypothesised that consumption of catechin-rich green tea would ameliorate postprandial hyperglycaemia and oxidative stress.

\section{Methods \\ Participants}

The present study was conducted according to the guidelines laid out in the Declaration of Helsinki, and all procedures involving human participants were approved by the Research Ethics Committees of the Tokyo Gakugei University (trial no. 2012-57). Written informed consent was obtained from all participants. The study participants consisted of Japanese postmenopausal women aged $62-73$ years $(n 22)$ who were randomly recruited from the general population of local communities. All the included participants had no difficulties with mobility or performing their daily activities. Participants with a history of CVD, those who used glucose-lowering medication in the previous 3 months, smokers, or women $<60$ years of age were excluded from the study. For the baseline evaluation, we administered a simple lifestyle-related questionnaire (physical activity, medication, sleep, alcohol intake and smoking). Participants were asked to maintain their daily diet and lifestyle during the study period.

\section{Anthropometry}

Body mass was measured to the nearest $0 \cdot 1 \mathrm{~kg}$ using a digital scale (InnerScan 50; Tanita Corporation). Height was measured to the nearest $0 \cdot 1 \mathrm{~cm}$ using a wall-mounted stadiometer (YS-OA; As One Corporation). BMI was calculated as weight $(\mathrm{kg})$ divided by the square of height $\left(\mathrm{m}^{2}\right)$. Waist circumference was measured to the nearest $0 \cdot 1 \mathrm{~cm}$ at the level of the umbilicus using a flexible plastic tape. Arterial blood pressure was measured from the right arm of the participants while in a seated position by using a standard mercury sphygmomanometer (605P; Yagami Company Limited). Participants were seated in a chair for $5 \mathrm{~min}$ before blood pressure was measured. At least two measurements were taken at each time point, and the mean of these values was recorded.

\section{Green tea and placebo beverage content}

Green tea and placebo beverages used in the present study were provided by Kao Corporation. A brewed green tea with a natural flavour was used as the placebo beverage, and catechin-rich green tea was prepared with a green tea extract and hot water. The green tea beverage contained $615 \mathrm{mg} / 350 \mathrm{ml}$ of total catechins $(33.2 \mathrm{mg}$ catechin, $22.9 \mathrm{mg}$ catechin gallate, $135.2 \mathrm{mg}$ gallocatechin, $108.4 \mathrm{mg}$ gallocatechin gallate, $34.8 \mathrm{mg}$ epicatechin, $39.9 \mathrm{mg}$ epicatechin gallate, $114.5 \mathrm{mg}$ epigallocatechin and $125.9 \mathrm{mg}$ epigallocatechin gallate) and $77.0 \mathrm{mg}$ caffeine. The placebo beverage contained $92 \mathrm{mg} / 350 \mathrm{ml}$ of total catechins $(5.7 \mathrm{mg}$ catechin, $3.0 \mathrm{mg}$ catechin gallate, $28.6 \mathrm{mg}$ gallocatechin, $18.0 \mathrm{mg}$ gallocatechin gallate, $4.1 \mathrm{mg}$ epicatechin, $3.8 \mathrm{mg}$ epicatechin gallate, $14.2 \mathrm{mg}$ epigallocatechin and $15.0 \mathrm{mg}$ epigallocatechin gallate) and $85.2 \mathrm{mg}$ caffeine. The green tea and placebo beverages were adjusted to contain the same levels of vitamin $\mathrm{C}$ and other minor polyphenols.

\section{Experimental protocol}

A double-blind, placebo-controlled, parallel design was used. The twenty-two postmenopausal women were randomly divided into the following two groups: placebo ( $\mathrm{P}, n$ 11) group and green tea (GT, $n$ 11) group. The physical characteristics of both groups are presented in Table 1. The participants of the GT and P groups were instructed to consume one bottle of their respective beverage per $\mathrm{d}$ with their breakfast for 4 weeks. Previous studies ${ }^{(20,21)}$ have reported that a 4 -week period of green tea ingestion can reduce fasting circulating concentrations of oxidative stress markers such as oxidised LDL and malondialdehyde.

At baseline and after 4 weeks, all participants visited the laboratory at 08.30 hours after a $10 \mathrm{~h}$ overnight fast (no intake of food or drink, except water). After a 15 min rest, a fasting venous blood sample was collected by venepuncture while participants were in a seated position. Participants then consumed a standardised breakfast. A clock was started when the participants began eating, and they were required to rest (e.g. read or write) in the laboratory for $6 \mathrm{~h}$ after the initiation of the breakfast meal. A second test meal (identical to the first meal) was consumed $3 \mathrm{~h}$ after the initiation of the first meal. Further venous blood samples were collected at 2, 4 and $6 \mathrm{~h}$ after the initiation of the breakfast meal.

\section{Test meals}

The test meals consisted of white bread, sliced cheese, yogurt, ham, mayonnaise, lettuce, tomato, butter and peanut butter. The meal was prescribed according to body mass; hence, we provided $1.00 \mathrm{~g}$ fat, $1.24 \mathrm{~g}$ carbohydrates, $0.52 \mathrm{~g}$ protein and $67 \mathrm{~kJ}$ energy per $\mathrm{kg}$ body mass. Energy derived was $56.3 \%$ from fat, $30 \cdot 8 \%$ from carbohydrate and $12.9 \%$ from protein. Previous studies ${ }^{(22,23)}$ have reported that this macronutrient composition increased postprandial oxidative stress. All participants were asked to consume the test meal within $20 \mathrm{~min}$. The time taken to consume the first postprandial test meal (i.e. at baseline) was recorded and replicated in the subsequent postprandial test meal (i.e. at week 4). None of the participants reported nausea or any gastrointestinal discomfort during or after each meal. Participants consumed either a placebo (catechins $92 \mathrm{mg} / 350 \mathrm{ml}$ ) or green tea (catechins 
Table 1. Changes in physical characteristics, green tea and coffee consumption at baseline and after 4 weeks (Mean values with their standard errors)

\begin{tabular}{|c|c|c|c|c|c|c|}
\hline & \multicolumn{2}{|c|}{$\begin{array}{l}\text { Placebo group } \\
\quad(n 11)\end{array}$} & \multicolumn{2}{|c|}{$\begin{array}{l}\text { Green tea group } \\
(n 11)\end{array}$} & \multirow[b]{2}{*}{$P$ (time) } & \multirow[b]{2}{*}{$P$ (interaction) } \\
\hline & Mean & SE & Mean & SE & & \\
\hline \multicolumn{7}{|l|}{ Age (years) } \\
\hline $\begin{array}{l}\text { Baseline } \\
4 \text { weeks }\end{array}$ & $66 \cdot 5$ & 0.6 & $66 \cdot 6$ & $1 \cdot 2$ & - & - \\
\hline \multicolumn{7}{|l|}{ Height (m) } \\
\hline $\begin{array}{l}\text { Baseline } \\
4 \text { weeks }\end{array}$ & 1.53 & 0.01 & 1.53 & 0.02 & - & - \\
\hline \multicolumn{7}{|c|}{ Body mass $(\mathrm{kg})$} \\
\hline Baseline & $55 \cdot 4$ & $2 \cdot 4$ & $55 \cdot 7$ & $2 \cdot 0$ & 0.001 & 0.843 \\
\hline 4 weeks & 53.9 & $2 \cdot 3$ & $54 \cdot 1$ & $1 \cdot 8$ & & \\
\hline \multicolumn{7}{|l|}{$\mathrm{BMI}\left(\mathrm{kg} / \mathrm{m}^{2}\right)$} \\
\hline Baseline & 23.4 & 0.8 & $23 \cdot 8$ & 0.8 & 0.001 & 0.811 \\
\hline 4 weeks & $22 \cdot 7$ & $0 \cdot 8$ & $23 \cdot 1$ & 0.8 & & \\
\hline \multicolumn{7}{|c|}{ Waist circumference $(\mathrm{cm})$} \\
\hline Baseline & $84 \cdot 7$ & $2 \cdot 3$ & 83.7 & $2 \cdot 4$ & 0.610 & 0.581 \\
\hline 4 weeks & $84 \cdot 8$ & $2 \cdot 4$ & $82 \cdot 3$ & $2 \cdot 2$ & & \\
\hline \multicolumn{7}{|c|}{ Systolic blood pressure $(\mathrm{mmHg})$} \\
\hline Baseline & 128 & 4 & 125 & 5 & 0.665 & 0.899 \\
\hline 4 weeks & 126 & 3 & 124 & 3 & & \\
\hline \multicolumn{7}{|c|}{ Diastolic blood pressure $(\mathrm{mmHg})$} \\
\hline Baseline & 81 & 2 & 77 & 2 & 0.001 & 0.652 \\
\hline 4 weeks & 76 & 1 & 71 & 2 & & \\
\hline \multicolumn{7}{|c|}{ Green tea consumption (cups/d) } \\
\hline Baseline & 4.4 & 0.4 & $3 \cdot 3$ & 0.3 & 0.679 & 0.492 \\
\hline 4 weeks & 3.9 & 0.4 & $3 \cdot 7$ & 0.3 & & \\
\hline \multicolumn{7}{|c|}{ Coffee consumption (cups/d) } \\
\hline Baseline & $1 \cdot 6$ & 0.2 & $2 \cdot 0$ & 0.3 & $0 \cdot 100$ & 0.566 \\
\hline 4 weeks & 2.0 & 0.2 & 1.3 & 0.1 & & \\
\hline
\end{tabular}

$615 \mathrm{mg} / 350 \mathrm{ml}$ ) beverage with breakfast. Participants consumed water ad libitum during the first postprandial testing, and the pattern and volume ingested was replicated in the subsequent postprandial testing.

\section{Standardisation of the diet and physical activity}

All participants weighed and recorded all food and drink consumed during the day before each postprandial testing period (i.e. at baseline and at week 4). All participants refrained from drinking alcohol $2 \mathrm{~d}$ before each postprandial testing, and they replicated their dietary intake during the day before each postprandial testing. Additionally, all participants were asked to remain inactive on the day before each postprandial testing and throughout each postprandial testing. For the determination of physical activity levels, all participants were instructed to wear a uniaxial accelerometer (Lifecorder EX; Suzuken Company Limited) during the experimental period (i.e. at 4 weeks). A number of studies ${ }^{(24,25)}$ have reported that the Lifecorder EX is considered as a validated accelerometer for evaluating and monitoring physical activity levels.

\section{Blood collection and analysis}

For measuring serum blood markers, samples were allowed to clot for $30 \mathrm{~min}$ at room temperature and then centrifuged at
$1300 \mathrm{~g}$ for $10 \mathrm{~min}$ at $4^{\circ} \mathrm{C}$. The serum sample was dispensed into plain microtubes and stored at $-80^{\circ} \mathrm{C}$ until the assay. For measuring plasma blood markers, blood samples collected into tubes containing EDTA were immediately centrifuged and stored at $-80^{\circ} \mathrm{C}$ until the assay. Plasma concentrations of total catechins, gallocatechin, gallocatechin gallate, epicatechin gallate, epigallocatechin and epigallocatechin gallate were measured by using HPLC with solid-phase extraction as described previously ${ }^{(26,27)}$. Enzymatic colorimetric assays were used to measure the plasma concentrations of glucose (GLU-HK (M); Shino-Test Corporation), serum TAG (PureAuto S TG-N; Sekisui Medical Company Limited) and NEFA (NEFA-HR; Wako Pure Chemical Industries Limited). Plasma concentrations of insulin were measured by ELISA. Serum concentrations of the derivatives of reactive oxygen metabolites (d-ROM) and biological antioxidant potential were measured using assay kits (Diacron). The d-ROM test measures the oxidative stress of blood samples by evaluating the level of reactive oxygen metabolites. This assay is based on the capability of $N, N$-diethyl- $p$-phenylenediamine to give a stable, coloured solution when it is transformed into its radical cation $\left(N, N \text {-diethyl- } p \text {-phenylenediamine }{ }^{+}\right)^{(28)}$. The biological antioxidant potential assay is a photometric test that determines the serum concentration of antioxidants capable of reducing Fe from the ferric to the ferrous form ${ }^{(29)}$. Plasma $\mathrm{H}_{2} \mathrm{O}_{2}$ concentration was measured using the Amplex Red reagent method (Molecular Probes, Invitrogen 
Detection Technologies). Concentrations of plasma thioredoxin (TRX; Immuno-Biological Laboratories Company Limited) were measured by ELISA.

\section{Statistical analysis}

Data were analysed using Predictive Analytics Software, version 18.0 for Windows (SPSS, Inc., IBM). Based on previous studies $^{(30,31)}$, we estimated the sample size of the present study (control ( $n$ 11) and exercise ( $n$ 11) groups). In fact, power analysis indicated that eleven subjects per group were required to obtain a large effect size (multi-way ANOVA, $>0.80)$ with an $80 \%$ power at $\alpha=0.05$. The effect size was calculated using the method outlined by Cohen ${ }^{(32)}$. The Kolmogorov-Smirnov test was used to check for the normality of the distribution of all the blood parameters. The distribution of these parameters did not differ significantly from normal values. Unpaired Student's $t$ tests were used to assess group differences between data acquired at baseline and at 4 weeks. Three-factor ANOVA with repeated measures was used to determine the effect of group (P or GT), intervention (baseline or week 4), and postprandial interval (0-6h) on the concentrations of blood markers. When significant interaction effects were detected, simple main-effects tests were employed. Statistical significance was accepted at the $5 \%$ level $(P<0.05)$. Results are presented as means with their standard errors.

\section{Results}

\section{Physiological characteristics and physical activity}

There were no differences in the physical and physiological characteristics between the groups at baseline and at week 4 (Table 1). No change was observed in the daily activity habits of the participants during the monitoring period (i.e. moderate to vigorous physical activity and step counts). During the study period, the moderate-to-vigorous physical activity level was $172 \cdot 4$ (SE 13.1) and 203.6 (SE 21.7) min/ week for the P and GT groups, respectively. The step counts were 7627 (sE 594) and 8226 (SE 633) steps/d for the P and GT groups, respectively. Both moderate-to-physical activity level and step counts did not differ between the groups.

\section{Fasting and postprandial catechins}

Plasma concentrations of total catechins, gallocatechin, gallocatechin gallate, epicatechin gallate, epigallocatechin

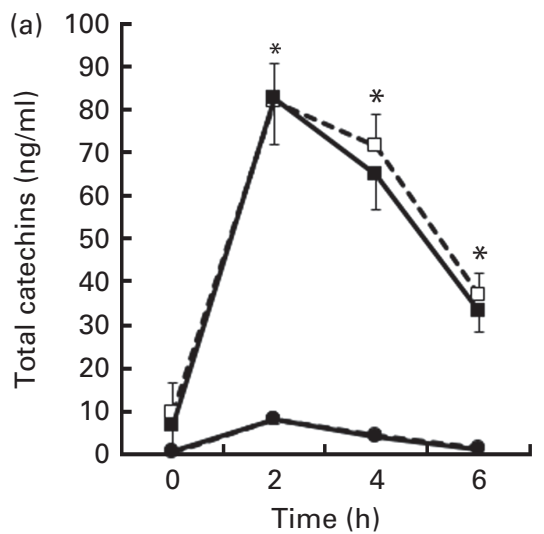

(d)

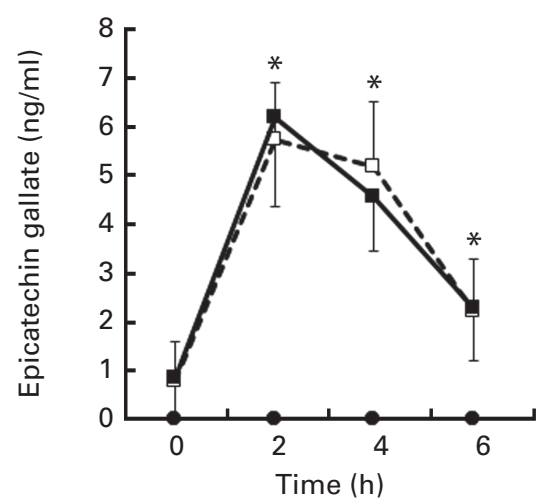

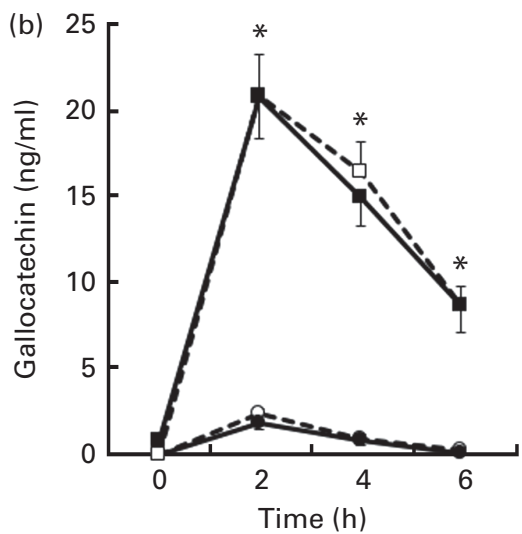

(e)

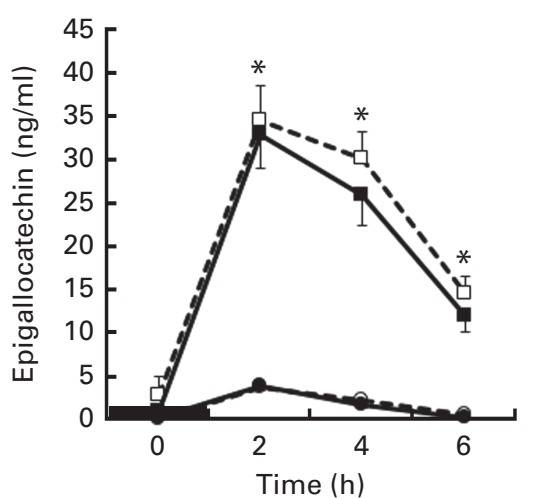

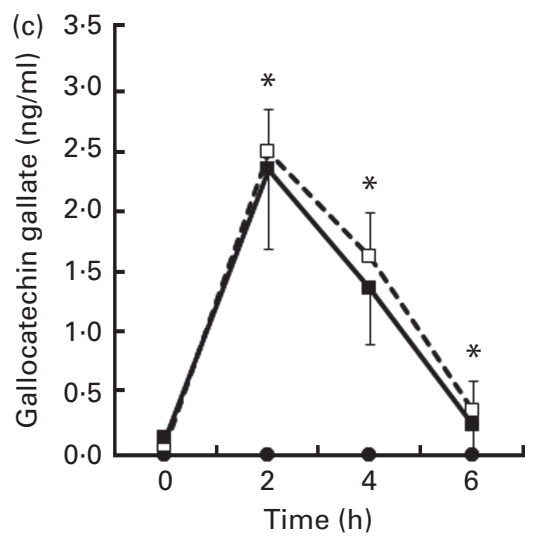

(f)

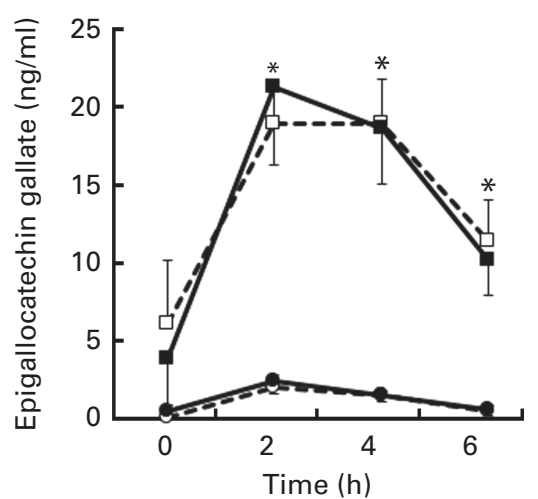

Fig. 1. Fasting and postprandial plasma concentrations of total catechins (a), gallocatechin (b), gallocatechin gallate (c), epicatechin gallate (d), epigallocatechin (e) and epigallocatechin gallate (f) in the placebo (P) and green tea (GT) groups at baseline and week 4. -O-, P baseline; - - -, P week 4; $-\square-$, GT baseline; - - -, GT week 4 . Values are means, with their standard errors represented by vertical bars. ${ }^{*}$ Mean value was significantly different from that of the $\mathrm{P}$ group $(P=0.001$; three-factor ANOVA). There were significant time $(P=0.001)$, group $(P=0.001)$ and time $\times$ group interaction effects $(P=0.001)$ observed for all the individual catechins (three-factor ANOVA). 

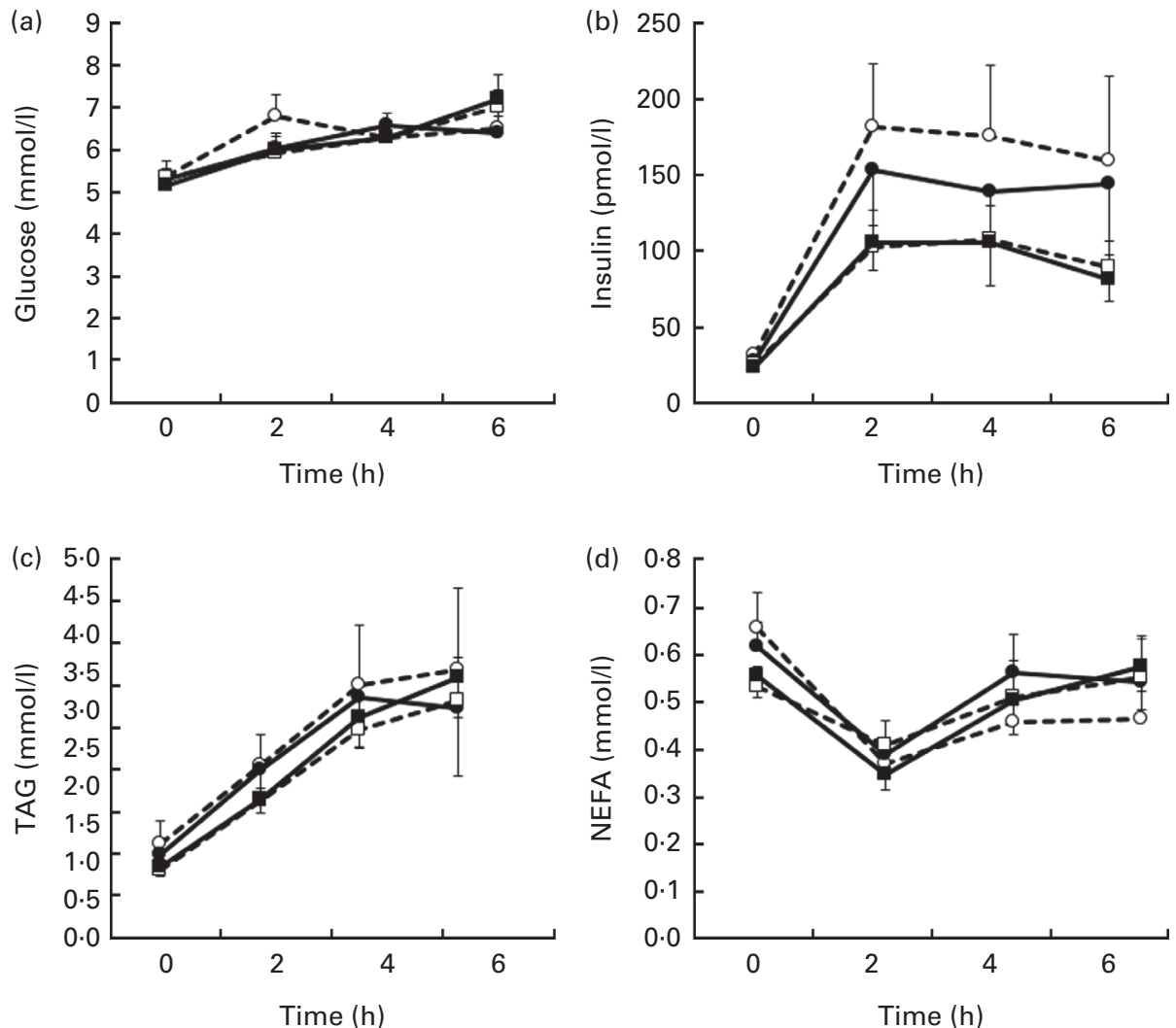

Fig. 2. Fasting and postprandial concentrations of plasma glucose (a), plasma insulin (b), serum TAG (c) and serum NEFA (d) in the placebo (P) and green tea (GT) groups at baseline and week 4. - $--_{-}$, P baseline; - - - P week 4; - $\square-$, GT baseline; - - -, GT week 4. Values are means, with their standard errors represented by vertical bars. There was a significant time effect $(P=0.001)$ observed for glucose, insulin, TAG and NEFA concentrations, and the time $\times$ group interaction was significant $(P=0.013)$ for glucose concentrations (three-factor ANOVA).

and epigallocatechin gallate are shown in Fig. 1(a)-(f). At the baseline level, there was no difference in all the individual catechins in the plasma between the groups. For all the individual catechins in the plasma, three-factor ANOVA with repeated measures showed a significant main effect of time $(P=0.001)$ and group $(P=0.001)$. Also, a significant time $\times$ group interaction $(P=0.001)$ was found for all the individual catechins in the plasma. The simple main-effects tests revealed that the concentrations of total catechins, gallocatechin, epigallocatechin gallate, epigallocatechin and epigallocatechin gallate were higher in the GT group than in the $\mathrm{P}$ group at 2,4 and $6 \mathrm{~h}$ after consumption of the meals (all time points; $P=0.001)$. The simple main-effects tests also revealed that gallocatechin gallate concentrations were higher in the GT group than in the $\mathrm{P}$ group at 2 and $4 \mathrm{~h}$ after consumption of the meals $(P=0 \cdot 001)$. The peak concentrations of all the individual catechins were observed at $2 \mathrm{~h}$ after consumption of the green tea beverage in the GT group. In addition, all the individual catechin concentrations did not significantly change in either group after 4 weeks compared with the baseline.

\section{Fasting and postprandial metabolites/hormones}

There were no differences in the values of all the blood parameters between the groups at baseline. Plasma glucose concentrations are shown in Fig. 2(a). Three-factor ANOVA with repeated measures showed a significant main effect of time $(P=0 \cdot 001)$ for plasma glucose concentrations. Significant interactions between time and group $(P=0.013)$ were also found. Conversely, plasma glucose concentrations did not significantly change in either group after 4 weeks compared with baseline. Plasma insulin concentrations are shown in Fig. 2(b). For plasma insulin concentrations, three-factor ANOVA with repeated measures showed a significant main effect of time $(P=0.001)$ and a tendency of a main effect of group $(P=0.055)$. For serum TAG and NEFA concentrations, threefactor ANOVA with repeated measures showed a significant main effect of time ( $P=0 \cdot 001)$ (Fig. 2(c) and (d)).

\section{Fasting and postprandial oxidative stress markers}

Serum d-ROM concentrations are shown in Fig. 3(a). Threefactor ANOVA with repeated measures showed a significant main effect of time $(P=0.001)$ for serum d-ROM concentrations. For plasma $\mathrm{H}_{2} \mathrm{O}_{2}$ and serum biological antioxidant potential concentrations, three-factor ANOVA with repeated measures showed a significant main effect of time $(P=0 \cdot 001$; Fig. 3(b) and (c)). Serum TRX concentrations are shown in Fig. 3(d). For serum TRX concentrations, three-factor ANOVA with repeated measures showed a significant main effect of time $(P=0.001)$ and a time $\times$ group interaction $(P=0 \cdot 012)$. The simple main-effects tests revealed that serum 

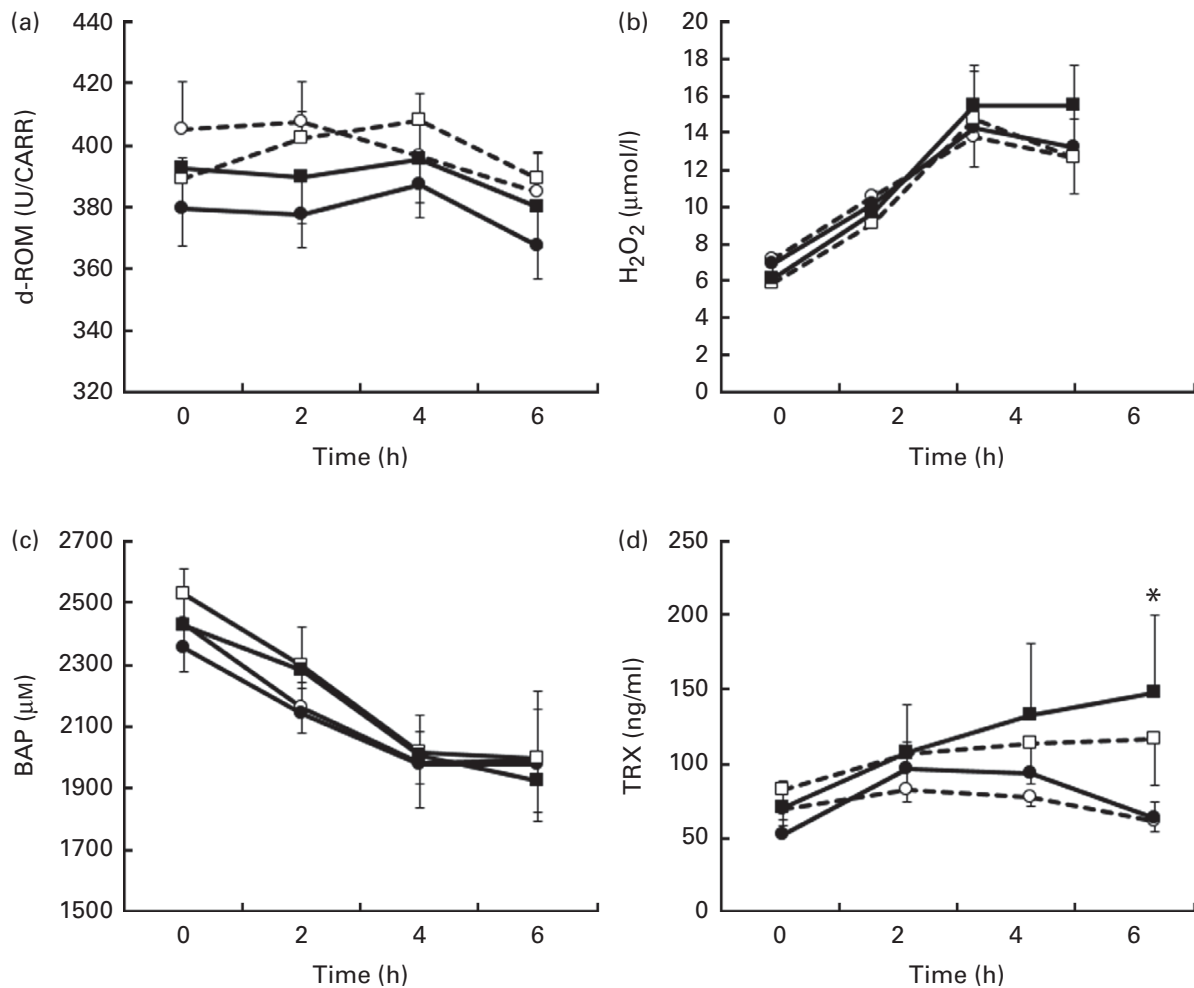

Fig. 3. Fasting and postprandial concentrations of serum derivatives of reactive oxygen metabolites (d-ROM; Carratelli units (U/CARR)) (a), plasma hydrogen peroxide (b), serum biological antioxidant potential (BAP) (c) and plasma thioredoxin (TRX) (d) in the placebo (P) and green tea (GT) groups at baseline and week 4.

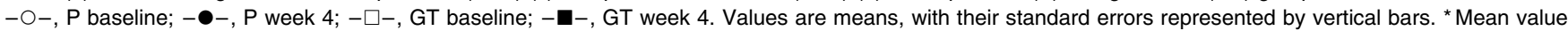
was significantly different from that of the $\mathrm{P}$ group $(P=0.030$; three-factor ANOVA). There was a significant time effect $(P=0.001)$ observed for $\mathrm{d}$-ROM, hydrogen peroxide, BAP and TRX concentrations, and the time $\times$ group interaction was significant $(P=0.012)$ for TRX concentrations (three-factor ANOVA).

TRX concentrations were higher in the GT group than in the $\mathrm{P}$ group at $6 \mathrm{~h}$ after consumption of the meals $(P=0 \cdot 030)$. However, serum TRX concentrations did not significantly change in either group after 4 weeks compared with baseline.

\section{Discussion}

To the best of our knowledge, the present study is the first to examine the effects of green tea intake on postprandial glucose and oxidative stress in postmenopausal women. The main finding of the present study is that an acute ingestion of catechin-rich green tea reduced postprandial plasma glucose concentrations in postmenopausal women. We also observed that an acute ingestion of catechin-rich green tea increased postprandial TRX concentrations. The present findings indicate that the intake of catechin-rich green tea improves postprandial hyperglycaemia and redox homeostasis in postmenopausal women. Conversely, there were no continuous effects associated with the ingestion of catechin-rich green tea on postprandial hyperglycaemia and oxidative stress.

Green tea is a rich source of polyphenol antioxidants called catechins, mainly epigallocatechin gallate, epigallocatechin, epicatechin gallate and epicatechin. Several studies ${ }^{(4,5,16,33)}$ have reported that green tea catechins possess antidiabetic properties and antioxidant capacity. However, few studies have measured blood catechin concentrations after drinking green tea for the evaluation of its antidiabetic and antioxidant effects. Thus, a major strength of the present study is that we directly measured blood catechin concentrations in response to an acute ingestion of catechin-rich green tea, and found a significant elevation in the plasma concentrations of total catechins, gallocatechin, gallocatechin gallate, epigallocatechin gallate, epigallocatechin and epigallocatechin gallate. Moreover, the peak concentration of all the individual catechins was observed at $2 \mathrm{~h}$ after ingestion of the green tea beverage in the GT group. These findings support a previous investigation where plasma catechin concentrations were elevated within $1-2 \mathrm{~h}$ after intake of green and black tea ${ }^{(34)}$.

In the present study, catechin-rich green tea attenuated postprandial plasma glucose concentrations in postmenopausal women. Moreover, postprandial insulin concentrations had a tendency to be lower in the GT group than in the $\mathrm{P}$ group. A previous investigation showed that the acute ingestion of green tea extract improved insulin sensitivity and glucose tolerance in young men ${ }^{(31)}$. Our data in postmenopausal women are consistent with that study. Green tea catechins have been reported to increase adipocyte insulin receptor binding and membrane GLUT4 protein content in rats $^{(35,36)}$. Another study has shown that epigallocatechin gallate enhances the expression of genes associated with insulin sensitivity ${ }^{(37)}$. Epigallocatechin gallate can also mimic insulin by increasing the tyrosine phosphorylation of both the insulin receptor and insulin receptor substrate-1, which 
stimulates glucose uptake. In addition to the enhancement of insulin sensitivity, the possible factor underlying the attenuation of postprandial glucose concentrations in the GT group may be the inhibition of intestinal $\alpha$-amylase, sucrose and $\alpha$-glucosidase, which reduce the absorption of carbohydrates from the intestine ${ }^{(38)}$. Therefore, green tea catechins have some biological activities that can possibly provide antidiabetic effects.

Another mechanism for improving glucose metabolism may be the improvement in oxidative stress including redox homeostasis ${ }^{(39)}$. It has been suggested that postprandial hyperglycaemia is positively correlated with the production of reactive oxygen species ${ }^{(10,40)}$. Indeed, ingestion of a highcarbohydrate or high-fat meal increases oxidative stress ${ }^{(11,22)}$. Particularly, intake of a high-fat meal results in greater postprandial oxidative stress than intake of a carbohydrate meal with the same energy content ${ }^{(11)}$. In the present study, serum d-ROM concentrations and plasma $\mathrm{H}_{2} \mathrm{O}_{2}$ of oxidative stress markers increased significantly following the ingestion of high-fat meals. Alternatively, both acute and continuous (i.e. for 4 weeks) intakes of green tea following high-fat meals have no impact on the levels of postprandial oxidative stress markers. Despite no overall difference in the levels of oxidative stress markers between the GT and P groups, we found that an acute ingestion of catechin-rich green tea increases postprandial TRX concentrations. TRX plays an essential role in cellular function and protection by limiting oxidative stress directly via its antioxidant capacity ${ }^{(41)}$. Further support for this finding is provided by other studies that have found that the TRX system was up-regulated via the nuclear factor E2-related factor 2 signalling pathway by the consumption of green tea components including epigallocatechin-3-gallate ${ }^{(42,43)}$. Previous studies ${ }^{(13,44)}$ have reported the relationship between glycaemic control disorders and oxidative stress. In diabetes mellitus, hyperglycaemia induces oxidative stress and contributes to the pathogenesis of diabetic vascular complications. Thus, elevated postprandial plasma TRX concentrations after an acute ingestion of catechin-rich green tea may have beneficial effects on oxidative stress status by mediating improved glucose metabolism in postmenopausal women.

Some investigators ${ }^{(16,32)}$ have reported that green tea catechins attenuated resting oxidative stress markers. However, in the present study, we observed that both acute and continuous (i.e. for 4 weeks) ingestion of green tea catechins did not influence resting (i.e. fasting) oxidative stress and antioxidant capacity. It is unclear as to why the ingestion of green tea catechins in the present study had no effects on resting oxidative stress and antioxidant capacity. Similar to the results of oxidative stress and antioxidant capacity markers, resting glucose and insulin concentrations at baseline and at week 4 were not significantly different between the groups. This inconsistency may be explained by the fact that most studies investigated the effects of green tea catechins on resting oxidative stress and antioxidant capacity markers for a long period of time (ranging from 8 weeks to 3 months) compared with the present study ${ }^{(15,16,45)}$. Thus, long-term ingestion of green tea may be effective in improving resting glucose and oxidative stress status.
Oxidative stress and redox status are determined by the balance between oxidant and antioxidant levels ${ }^{(46)}$. It is important to note that oxidative stress markers and antioxidant capacity, which we measured in the blood, do not represent oxidative stress status in the cells. However, some studies ${ }^{(47,48)}$ have reported that blood oxidative stress markers are associated with oxidative stress status in the tissues and cells. Moreover, there are several factors that influence and regulate redox status ${ }^{(49,50)}$. Therefore, we paid careful attention to several factors, including the nutritional contents of the beverage such as antioxidants (i.e. vitamin $\mathrm{C}$ and vitamin E) and the participants' physical activity (i.e. moderate to vigorous physical activity and step counts), medications, environmental factors and genetic factors. We can minimise these effects on blood redox status by setting up the exception criteria. Although we evaluated redox homeostasis, which was determined from serum TRX concentrations, the TRX system is a major antioxidant system that promotes the reduction of proteins by the cysteine thiol-disulphide exchange, which plays a vital role in maintaining the cellular redox balance ${ }^{(41,45)}$. Furthermore, to our knowledge, no information is available regarding the effect of green tea catechins on postprandial TRX concentrations in human subjects. Additional research is required to assess other redox parameters.

In conclusion, the present study demonstrates that an acute ingestion of catechin-rich green tea can decrease postprandial plasma glucose concentrations in postmenopausal women. This improvement may be explained by the fact that green tea ingestion elevated postprandial TRX concentrations, which suggests improved postprandial redox status, including TRX concentrations. However, continuous ingestion of catechin-rich green tea for 4 weeks showed no additive effects on the improvement of postprandial hyperglycaemia and oxidative stress in postmenopausal women.

\section{Acknowledgements}

The present study was funded by Kao Corporation, Tokyo, Japan.

The authors' contributions are as follows: M. Takahashi deigned the study, supervised the data collection, performed the blood and data analysis, and drafted the manuscript; M. M. conceived the study, obtained funding, recruited the participants, designed the study, assisted M. Takahashi with data collection and edited the manuscript; K. S. performed the venous blood collection and provided guidance and assistance to M. Takahashi during the study; S. B. and H.-K. K. assisted M. Takahashi with physiological and blood analysis; T. W., M. Takeshita and K. Y. designed the study, prepared the test beverages and provided guidance and assistance to M. Takahashi during the study; Y. M. designed the study, performed the blood catechin analysis in a double-blind manner, and provided guidance and assistance to M. Takahashi during the study. All authors read and approved the final manuscript.

Conflicts of interest: T. W., Y. M., M. Takeshita and K. Y. are employees of Kao Corporation. M. M. received a research grant from Kao Corporation. T. W., Y. M., M. Takeshita and 
K. Y. were not involved in the interpretation of the results. The rest of the authors have no conflicts of interest to declare.

\section{References}

1. Kuriyama S (2008) The relation between green tea consumption and cardiovascular disease as evidenced by epidemiological studies. J Nutr 138, 1548S-1553S.

2. Kuriyama S, Shimazu T, Ohmori K, et al. (2006) Green tea consumption and mortality due to cardiovascular disease, cancer, and all causes in Japan: the Ohsaki study. JAMA 296, 1255-1265.

3. Suzuki E, Yorifuji T, Takao S, et al. (2009) Green tea consumption and mortality among Japanese elderly people: the prospective Shizuoka elderly cohort. Ann Epidemiol 19, $732-739$

4. Zheng XX, Xu YL, Li SH, et al. (2013) Effects of green tea catechins with or without caffeine on glycemic control in adults: a meta-analysis of randomized controlled trials. $\mathrm{Am}$ J Clin Nutr 97, 750-762.

5. Liu K, Zhou R, Wang B, et al. (2013) Effect of green tea on glucose control and insulin sensitivity: a meta-analysis of 17 randomized controlled trials. Am J Clin Nutr 98, $340-348$.

6. Anonymous (1999) Glucose tolerance and mortality: comparison of WHO and American Diabetes Association diagnostic criteria. The DECODE study group. European Diabetes Epidemiology Group. Diabetes Epidemiology: Collaborative analysis of Diagnostic criteria in Europe. Lancet 354, 617-621.

7. Nakagami T \& DECODA Study Group (2004) Hyperglycaemia and mortality from all causes and from cardiovascular disease in five populations of Asian origin. Diabetologia 47, 385-394

8. Bae JH, Bassenge E, Kim KB, et al. (2001) Postprandial hypertriglyceridemia impairs endothelial function by enhanced oxidant stress. Atherosclerosis 155, 517-523.

9. Monnier L, Mas E, Ginet C, et al. (2006) Activation of oxidative stress by acute glucose fluctuations compared with sustained chronic hyperglycemia in patients with type 2 diabetes. JAMA 295, 1681-1687.

10. Sies H, Stahl W \& Sevanian A (2005) Nutritional, dietary and postprandial oxidative stress. J Nutr 135, 969-972.

11. Bloomer RJ, Kabir MM, Marshall KE, et al. (2010) Postprandial oxidative stress in response to dextrose and lipid meals of differing size. Lipids Health Dis 9, 79.

12. Wallace JP, Johnson B, Padilla J, et al. (2010) Postprandial lipaemia, oxidative stress and endothelial function: a review. Int J Clin Pract 64, 389-403.

13. Neri S, Calvagno S, Mauceri B, et al. (2010) Effects of antioxidants on postprandial oxidative stress and endothelial dysfunction in subjects with impaired glucose tolerance and type 2 diabetes. Eur J Nutr 49, 409-416.

14. Koutelidakis AE, Rallidis L, Koniari K, et al. (2013) Effect of green tea on postprandial antioxidant capacity, serum lipids, C-reactive protein and glucose levels in patients with coronary artery disease. Eur J Nutr 53, 479-486.

15. Bloomer RJ, Trepanowski JF \& Farney TM (2013) Influence of acute coffee consumption on postprandial oxidative stress. Nutr Metab Insights 6, 35-42.

16. Bogdanski P, Suliburska J, Szulinska M, et al. (2012) Green tea extract reduces blood pressure, inflammatory biomarkers, and oxidative stress and improves parameters associated with insulin resistance in obese, hypertensive patients. Nutr Res 32, 421-427.
17. Erba D, Riso P, Bordoni A, et al. (2005) Effectiveness of moderate green tea consumption on antioxidative status and plasma lipid profile in humans. J Nutr Biochem 16, 144-149.

18. Panza VS, Wazlawik E, Ricardo Schutz G, et al. (2008) Consumption of green tea favorably affects oxidative stress markers in weight-trained men. Nutrition 24, 433-442.

19. Mecocci P, Fano G, Fulle S, et al. (1999) Age-dependent increases in oxidative damage to DNA, lipids, and proteins in human skeletal muscle. Free Radic Biol Med 26, 303-308

20. Inami S, Takano M, Yamamoto M, et al. (2007) Tea catechin consumption reduces circulating oxidized low-density lipoprotein. Int Heart J 48, 725-732.

21. Jowko E, Sacharuk J, Balasinska B, et al. (2011) Green tea extract supplementation gives protection against exercise-induced oxidative damage in healthy men. Nutr Res 31, 813-821.

22. Bloomer RJ, Ferebee DE, Fisher-Wellman KH, et al. (2009) Postprandial oxidative stress: influence of sex and exercise training status. Med Sci Sports Exerc 41, 2111-2119.

23. Tsai WC, Li YH, Lin CC, et al. (2004) Effects of oxidative stress on endothelial function after a high-fat meal. Clin Sci (Lond) 106, 315-319.

24. Ayabe M, Kumahara H, Morimura K, et al. (2013) Accumulation of short bouts of non-exercise daily physical activity is associated with lower visceral fat in Japanese female adults. Inter I Sports Med 34, 62-67.

25. Kumahara H, Schutz Y, Ayabe M, et al. (2004) The use of uniaxial accelerometry for the assessment of physical-activityrelated energy expenditure: a validation study against whole-body indirect calorimetry. Br J Nutr 91, 235-243.

26. Unno T, Sagesaka YM \& Kakuda T (2005) Analysis of tea catechins in human plasma by high-performance liquid chromatography with solid-phase extraction. J Agric Food Chem 53, 9885-9889.

27. Umegaki K, Sugisawa A, Yamada K, et al. (2001) Analytical method of measuring tea catechins in human plasma by solid-phase extraction and HPLC with electrochemical detection. J Nutr Sci Vitaminol (Tokyo) 47, 402-408.

28. Cornelli U, Terranova R, Luca S, et al. (2001) Bioavailability and antioxidant activity of some food supplements in men and women using the D-Roms test as a marker of oxidative stress. J Nutr 131, 3208-3211.

29. Pasquini A, Luchetti E, Marchetti V, et al. (2008) Analytical performances of d-ROMs test and BAP test in canine plasma. Definition of the normal range in healthy Labrador dogs. Vet Res Commun 32, 137-143.

30. Narotzki B, Reznick AZ, Navot-Mintzer D, et al. (2013) Green tea and vitamin $\mathrm{E}$ enhance exercise-induced benefits in body composition, glucose homeostasis, and antioxidant status in elderly men and women. J Am Coll Nutr 32, 31-40.

31. Venables MC, Hulston CJ, Cox HR, et al. (2008) Green tea extract ingestion, fat oxidation, and glucose tolerance in healthy humans. Am J Clin Nutr 87, 778-784

32. Cohen J (1988) Statistical Power Analysis for the Behavioral Sciences, 2nd ed. Hillsdale, NJ: Lawrence Erlbaum Associates, Inc.

33. Suliburska J, Bogdanski P, Szulinska M, et al. (2012) Effects of green tea supplementation on elements, total antioxidants, lipids, and glucose values in the serum of obese patients. Biol Trace Elem Res 149, 315-322.

34. Rietveld A \& Wiseman S (2003) Antioxidant effects of tea: evidence from human clinical trials. J Nutr 133, 3285S-3292S.

35. Wu LY, Juan CC, Ho LT, et al. (2004) Effect of green tea supplementation on insulin sensitivity in Sprague-Dawley rats J Agric Food Chem 52, 643-648.

36. Wu LY, Juan CC, Hwang LS, et al. (2004) Green tea supplementation ameliorates insulin resistance and increases 
glucose transporter IV content in a fructose-fed rat model. Eur J Nutr 43, 116-124.

37. Sakurai N, Mochizuki K, Kameji H, et al. (2009) (-)-Epigallocatechin gallate enhances the expression of genes related to insulin sensitivity and adipocyte differentiation in 3T3-L1 adipocytes at an early stage of differentiation. Nutrition $\mathbf{2 5}$, 1047-1056.

38. Collins QF, Liu HY, Pi J, et al. (2007) Epigallocatechin-3-gallate (EGCG), a green tea polyphenol, suppresses hepatic gluconeogenesis through $5^{\prime}$-AMP-activated protein kinase. J Biol Chem 282, 30143-30149.

39. Roghani M \& Baluchnejadmojarad T (2010) Hypoglycemic and hypolipidemic effect and antioxidant activity of chronic epigallocatechin-gallate in streptozotocin-diabetic rats. Pathophysiology 17, 55-59.

40. Ceriello A, Taboga C, Tonutti L, et al. (2002) Evidence for an independent and cumulative effect of postprandial hypertriglyceridemia and hyperglycemia on endothelial dysfunction and oxidative stress generation: effects of short- and longterm simvastatin treatment. Circulation 106, 1211-1218.

41. Lappalainen Z, Lappalainen J, Oksala NK, et al. (2009) Diabetes impairs exercise training-associated thioredoxin response and glutathione status in rat brain. J Appl Physiol (1985) 106, 461-467.

42. Tanito M, Agbaga MP \& Anderson RE (2007) Upregulation of thioredoxin system via Nrf2-antioxidant responsive element pathway in adaptive-retinal neuroprotection in vivo and in vitro. Free Radic Biol Med 42, 1838-1850.
43. Sahin K, Tuzcu M, Gencoglu H, et al. (2010) Epigallocatechin-3-gallate activates Nrf2/HO-1 signaling pathway in cisplatin-induced nephrotoxicity in rats. Life Sci $\mathbf{8 7}$, $240-245$.

44. Ceriello A, Esposito K, Piconi L, et al. (2008) Oscillating glucose is more deleterious to endothelial function and oxidative stress than mean glucose in normal and type 2 diabetic patients. Diabetes 57, 1349-1354.

45. Wu AH, Spicer D, Stanczyk FZ, et al. (2012) Effect of 2-month controlled green tea intervention on lipoprotein cholesterol, glucose, and hormone levels in healthy postmenopausal women. Cancer Prev Res 5, 393-402.

46. Berndt C, Lillig CH \& Holmgren A (2007) Thiol-based mechanisms of the thioredoxin and glutaredoxin systems: implications for diseases in the cardiovascular system. $A m \mathrm{~J}$ Physiol Heart Circ Physiol 292, H1227-H1236.

47. Veskoukis AS, Nikolaidis MG, Kyparos A, et al. (2009) Blood reflects tissue oxidative stress depending on biomarker and tissue studied. Free Radic Biol Med 47, 1371-1374.

48. Gil L, Siems W, Mazurek B, et al. (2006) Age-associated analysis of oxidative stress parameters in human plasma and erythrocytes. Free Radic Res 40, 495-505.

49. Dato S, Crocco P, D'Aquila P, et al. (2013) Exploring the role of genetic variability and lifestyle in oxidative stress response for healthy aging and longevity. Int $J$ Mol Sci 14, $16443-16472$.

50. Bocci V \& Valacchi G (2013) Free radicals and antioxidants: how to reestablish redox homeostasis in chronic diseases? Curr Med Chem 20, 3397-3415. 